\title{
THE MEASUREMENT OF TROPOSPHERIC TEMPERATURE PROFILES USING RAYLEIGH-BRILLOUIN SCATTERING: RESULTS FROM LABORATORY AND ATMOSPHERIC STUDIES
}

\author{
Benjamin Witschas $^{1 *}$, Oliver Reitebuch ${ }^{1}$, Christian Lemmerz ${ }^{1}$, Pau Gomez Kableka ${ }^{1}$, Sergey \\ Kondratyev ${ }^{2}$, Ziyu $\mathbf{G u}^{3}$, Wim Ubachs ${ }^{3}$ \\ ${ }^{I}$ German Aerospace Center (DLR), Institute of Atmospheric Physics, 82234 Oberpfaffenhofen, Germany \\ ${ }^{2}$ Angstrom Ltd., 630090 Inzhenernaya16, Novosibirsk, Russia \\ ${ }^{3}$ Department of Physics and Astronomy, LaserLaB, VU University, 1081 HV Amsterdam, Netherlands
}

*Email:Benjamin.Witschas@dlr.de

\begin{abstract}
In this letter, we suggest a new method for measuring tropospheric temperature profiles using Rayleigh-Brillouin (RB) scattering. We report on laboratory $\mathrm{RB}$ scattering measurements in air, demonstrating that temperature can be retrieved from RB spectra with an absolute accuracy of better than $2 \mathrm{~K}$. In addition, we show temperature profiles from $2 \mathrm{~km}$ to $15.3 \mathrm{~km}$ derived from RB spectra, measured with a high spectral resolution lidar during daytime. A comparison with radiosonde temperature measurements shows reasonable agreement. In cloud-free conditions, the temperature difference reaches up to $5 \mathrm{~K}$ within the boundary layer, and is smaller than $2.5 \mathrm{~K}$ above. The statistical error of the derived temperatures is between $0.15 \mathrm{~K}$ and $1.5 \mathrm{~K}$.
\end{abstract}

\section{INTRODUCTION}

Temperature is a key parameter of the state of the atmosphere and hence, temperature data play an important role in atmospheric dynamics, climatology, meteorology, and chemistry. For this reason, observational data on atmospheric temperature profiles are important inputs to models predicting the atmospheric state and to retrieve details of other atmospheric properties, such as wind, pressure, relative humidity, or other trace gas concentrations.

At present, lidar instruments enable the measurement of temperature with high accuracy $(\approx 1 \mathrm{~K})$, high vertical resolution $(\approx 100 \mathrm{~m})$, and long range (from ground up to $105 \mathrm{~km}$ ) [1]. In order to achieve this performance, it is common to use integration technique lidar systems, fluorescence lidars, or rotational Raman lidars. The former two are only applicable to stratospheric or mesospheric measurements because they can only be applied in air masses that do not contain aerosols, or where appropriate fluorescing metal atoms exist. In contrast, Raman lidars can be applied for tropospheric measurements [2]. Nevertheless, it has to be mentioned that the Raman scattering cross section is quite low. Thus, powerful lasers, sophisticated background filters, or night-time operation are required to obtain reliable results. In particular, the rotational Raman differential backscattering cross section (considering Stokes and anti-Stokes branches) is about a factor of 50 smaller than the one of Rayleigh scattering [3]. Regarding this, it is a preferred method to retrieve temperatures from Rayleigh-Brillouin (RB) scattered light, as it can be done by high spectral resolution lidars (HSRL). In particular, atmospheric temperature can be derived by resolving the RB spectrum with high spectral resolution filters, such as atomic vapor cells [4] or Fabry-Pérot interferometers [5], and relating the measured spectrum to an appropriate line shape model. Thus, apart from the challenging optical setup, such an approach is depending on the detailed knowledge of the RB scattering process and on the accuracy of line shape models that describe the spectral distribution of RB scattered light. In 1974, Tenti et al. [6] developed a model the so-called Tenti S6 model - for describing RB spectra of light scattered in molecular gases of single species, which is since then considered as the best model available. Recently performed laboratory studies [7-8] showed that the Tenti S6 model describes the measured RB spectra in air with deviations smaller than $2 \%$ if modifications on the model calculations are performed taking 
into account the mixed nature of air as scattering medium. In order to retrieve temperature from measured RB profiles, they have to be analyzed with the Tenti S6 model in a certain optimization procedure as for instance a least-squares fit with temperature as free fitting parameter. As for such method it is an important issue how the $2 \%$ model deviation transfers into a temperature error.

So as to deal with this issue, RB scattering measurements in air at a wavelength of $403 \mathrm{~nm}$, at a scattering angle of $91.7^{\circ}$, for temperatures from $257 \mathrm{~K}$ to $330 \mathrm{~K}$ and pressures from $871 \mathrm{hPa}$ to $1013 \mathrm{hPa}$ were performed and used to verify a temperature retrieval algorithm. The results from this study are discussed in section 2 . In addition, atmospheric daytime measurements of tropospheric temperature profiles $(2-15 \mathrm{~km})$ using RB scattering were performed demonstrating that temperatures can be derived from RB profiles with an accuracy better than $2 \mathrm{~K}$ (see section 3 ). Based on the results from the laboratory and atmospheric studies, a novel lidar temperature receiver is suggested and is currently under development at the German Aerospace center DLR (see section 4). It is based on a Fizeau interferometer used to resolve the RB spectrum without any scanning procedures.

\section{LABORATORY MEASUREMENTS}

In order to investigate the principal accuracy that can be reached by deriving temperature from RB spectra in air, highly accurate laboratory measurements were performed at the LaserLaB of VU University Amsterdam. Details about the experimental setup and the applied retrieval algorithm can be found in [9]. An example of measured RB profiles at different temperatures and pressures can be seen in Figure 1 (top). The spectra illustrate the high signal-to-noise ratio on the measured RB spectra obtained, as well as the line shape broadening with increasing temperature.

In Figure 1 (bottom), the retrieved temperatures $T_{\text {model }}$ are shown as a function of the reference temperature $T_{P t 100}$. It is obvious that there is very good accordance between retrieved- and reference temperature. In particular, the absolute difference is less than $2 \mathrm{~K}$ for all measurements. A linear correlation (Figure 1, blue dashed lines) yields a slope of $1.01 \pm 0.02$, and thus clearly demonstrates the possibility of retrieving absolute temperatures from $\mathrm{RB}$ profiles with high accuracy.
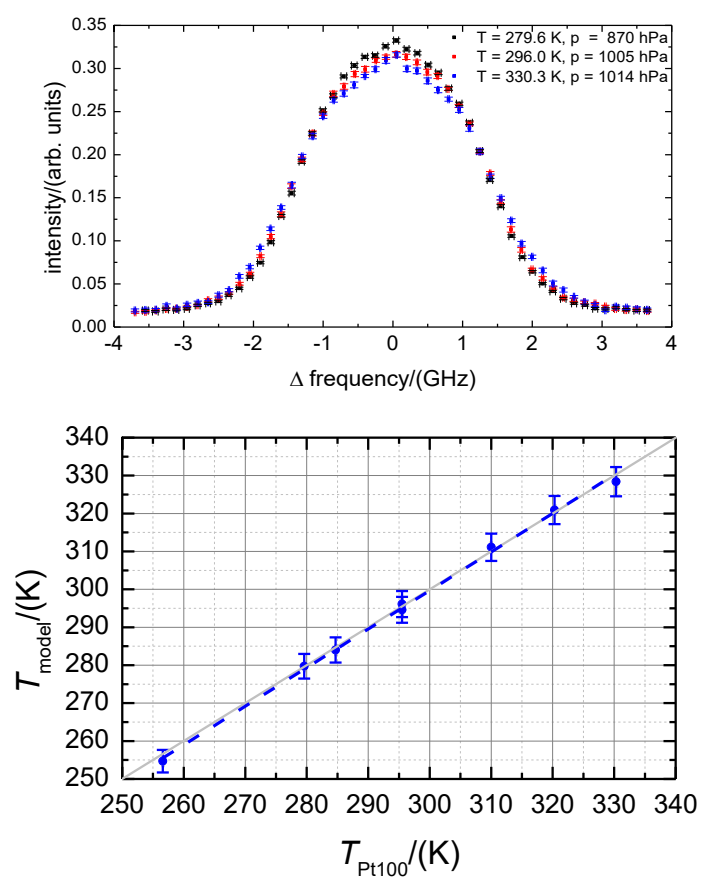

Figure 1: (top): RB spectra measured at $403 \mathrm{~nm}$, a scattering angle of $91.7^{\circ}$ and different temperatures (colors). (bottom): Temperatures derived from measured spectra by using the Tenti S6 model (solid blue dots) and the corresponding linear fit (dashed blue line). The gray line represents the $T_{\text {model }}=T_{P t 100}$ line.

\section{ATMOSPHERIC MEASUREMENTS}

In addition to the laboratory measurements, atmospheric measurements have been performed. The lidar used for this study was not originally built for this purpose, but is actually a Doppler wind lidar, used for validating the instrument of the ADM-Aeolus satellite mission initiated by ESA [10]. However, as it is shown in [11], the instrument is also suited for measuring temperature by means of RB scattering.

To record RB profiles, the frequency of the laser is changed in defined steps $\Delta \mathrm{f}$ (e.g., $\Delta \mathrm{f}=125$ $\mathrm{MHz}$ ), over a range of $12 \mathrm{GHz}$ (at $354.89 \mathrm{~nm}$ ) and a Fabry-Perot interferometer (Full Width Half Maximum (FWHM) $1.7 \mathrm{GHz}$, Free Spectral Range (FSR) 11 GHz) is used as frequency discriminator. Considering 90 steps and duration of $18 \mathrm{~s}$ per step, the sampling of the entire RB line 
shape requires $28 \mathrm{~min}$. It is worth mentioning that sampling the RB spectrum with only a few points $(\approx 15)$ would be enough to reach maximum accuracy for the temperature retrieval (according to simulations). However, as the measurement uncertainty is inversely proportional to the signal level, reducing the sample number or rather measurement time increases the uncertainty of the derived temperatures. Furthermore, it is beneficial to have more samples to resolve the peak resulting from particle scattering in aerosol-rich regions. An example of measured $\mathrm{RB}$ line shapes $(\Delta \mathrm{f}=$ $250 \mathrm{MHz})$ at different distances $(6.4 \mathrm{~km}-12.7 \mathrm{~km})$ is shown in Figure 2 (top). It is pointed out that the signal from bin $21(10.2 \mathrm{~km}-11.5 \mathrm{~km})$ shows a remarkable contribution from significantly narrower particle scattering on a cirrus cloud, which also has to be considered for temperature retrieval.

A generic temperature profile retrieved from the measured RB spectra is shown in Figure 2 (bottom, left, red dots). For this measurement, the corresponding RB profile was sampled with 93 points separated by $125 \mathrm{MHz}$, leading to a frequency range of about $11.6 \mathrm{GHz}$ and a measurement time of $28 \mathrm{~min}$. The range gate size was vertically adapted between $0.3 \mathrm{~km}$ and $2.2 \mathrm{~km}$ to keep the number of received photons large enough to minimize the statistical error. The measured temperature profile is compared with radiosonde temperatures (Figure 2, bottom, left, black line), launched during the lidar measurement about $27 \mathrm{~km}$ away from the lidar. The statistical error of the obtained temperature values (Figure 2, bottom, left, error bars) is calculated by applying a maximum likelihood estimator and considering solely Poisson noise on the measured data points (details can be found in [11]).

Although the temperature measurements were performed during noon, they show good agreement to radiosonde measurements. The profiles range from 2 to $15.3 \mathrm{~km}$, and temperature difference reaches up to $5 \mathrm{~K}$ within the boundary layer and is smaller than $2.5 \mathrm{~K}$ above. The inversion layer at $3 \mathrm{~km}$ is obvious. The statistical error varies from $0.15 \mathrm{~K}$ (at $2 \mathrm{~km}$ ) to $1.5 \mathrm{~K}$ (at $15 \mathrm{~km})$. However, it has to be mentioned that other measurements showed contamination by particle scattering that led to temperature errors of several Kelvin [14]. Although the influence of particle scattering was considered within the temperature retrieval, uncertainties are caused by temporal variations in the particle load during line shape sampling. Simulations confirm that discrepancies of larger than $20 \mathrm{~K}$ can occur if the particle scattering is not constant within the sampling process. In addition, it has to be pointed out that the limited FPI resolution of $1.7 \mathrm{GHz}$ is not perfectly suited to resolving the influence of particle scattering on the RB spectrum.

Thus, it is concluded that, although very promising for clear air conditions, the suggested approach still suffers from the influence of particle scattering.
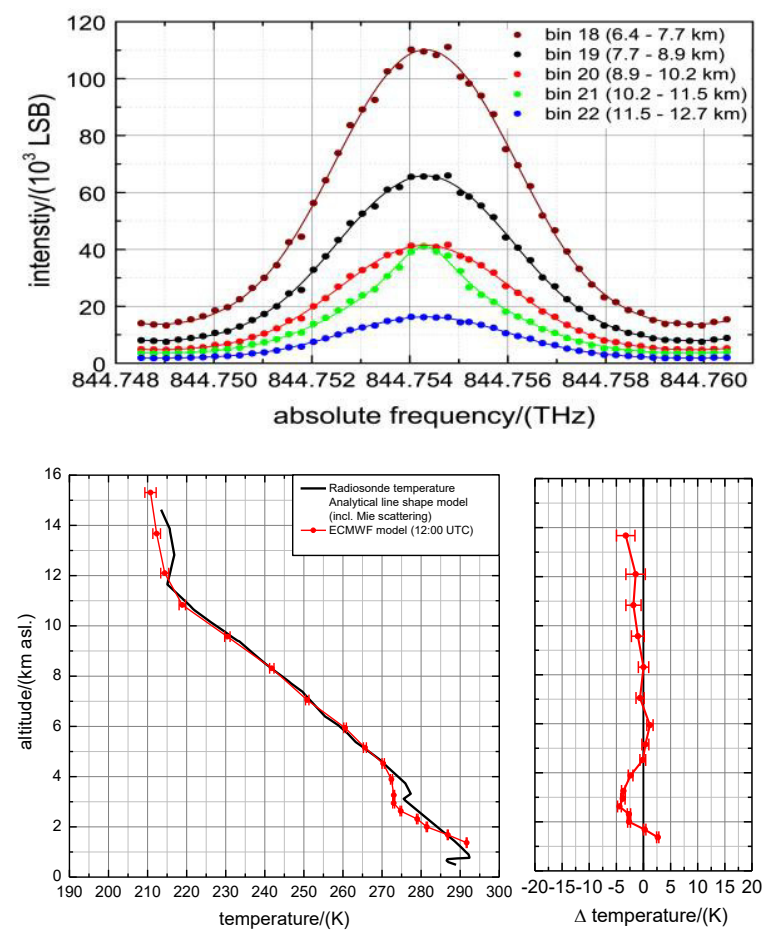

Figure 2: (top): Measured RB line shapes for different distances from the lidar (dots) and best-fits according to the Tenti S6 model (lines). (bottom, left): Temperature profiles derived from lidar measurements (red dots) compared with temperatures measured by radiosonde (black line). (bottom, right): Difference between radiosonde and lidar temperature values.

\section{OUTLOOK}

To further reduce the measurement time for one temperature profile, an instrument that is able to sample the entire RB line shape simultaneously without any scanning procedures is proposed. This can be realized by imaging techniques such as, for instance, resolving the RB spectrum with a 
Fizeau interferometer and imaging the throughput on a multichannel PMT array. By using such a detector, even a varying aerosol load would not negatively influence the temperature retrieval. Furthermore, by designing the Fizeau interferometer with sufficiently high resolution, the aerosol peak can be better distinguished from the RB spectrum, which will additionally improve the temperature retrieval in regions of larger particle scattering. In addition to that, it is feasible to design a hybrid system to measure temperature profiles that uses both Raman scattering in cloudy conditions and RB scattering during daytime and cloudless conditions.

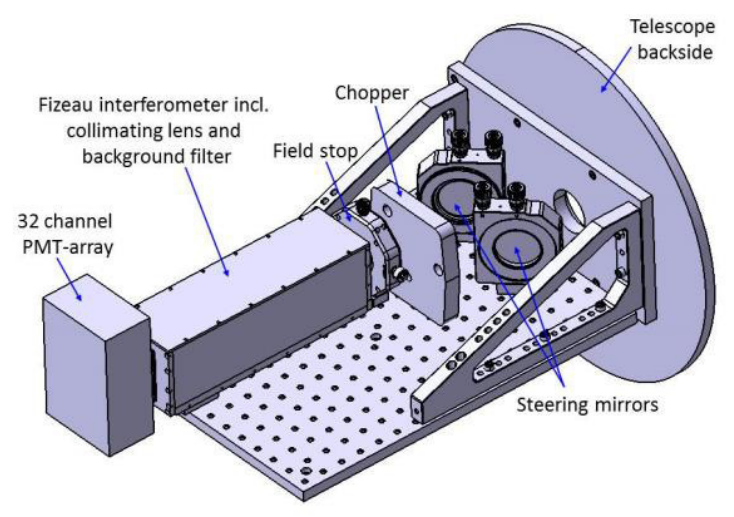

Figure 3: Sketch of a new lidar temperature receiver currently under development at DLR

Such kind of novel temperature receiver is currently under development at DLR and first atmospheric measurements are foreseen for mid of 2015. A sketch of the temperature receiver is shown in Figure 3.

\section{ACKNOWLEDGEMENT}

This work was partly funded by DLR and ESA within the Network Partnering Initiative, Contract No. 4000104130. The valuable suggestions of A.G. Straume (ESA) and the technical support of E. Nagel (DLR) are highly appreciated.

\section{REFERENCES}

[1] Alpers M., R. Eixmann, C. Fricke-Begemann, M. Greding, and J. Höffner, 2004: Temperature lidar measurements from 1 to $105 \mathrm{~km}$ altitude using resonance, Rayleigh, and Rotational Raman scattering, Atmos. Chem. Phys. 4, 793-800.
[2] Behrendt A., T. Nakamura, T. Tsuda, 2004: Combined temperature lidar for measurements in the troposphere, stratosphere, and mesosphere, Appl. Opt. 43, 2930-2939.

[3] Witschas B. 2012: Light Scattering on Molecules in the Atmosphere, in Atmospheric Physics: Background - Methods - Trends, U. Schumann, ed. (Springer Berlin Heidelberg, 2012), pp. 69-83.

[4] Hair J., L. Caldwell, D. Krueger, and C. She, 2001: High-Spectral-Resolution Lidar with Iodine-Vapor Filters: Measurement of Atmospheric-State and Aerosol Profiles, Appl. Opt. 40, 5280-5294.

[5] Hua D., M. Uchida, and T. Kobayashi, 2005: Ultraviolet Rayleigh-Mie lidar for daytimetemperature profiling of the troposphere, Appl. Opt. 44, 1315-1322.

[6] Tenti G., C. Boley, and R. Desai, 1974: On the kinetic model description of Rayleigh-Brillouin scattering from molecular gases, Can. J. Phys. 52, 285-290.

[7] Witschas B., M. O. Vieitez, E.-J. van Duijn, O. Reitebuch, W. van de Water, and W. Ubachs, 2010: Spontaneous Rayleigh-Brillouin scattering of ultraviolet light in nitrogen, dry air, and moist air, Appl. Opt. 49, 4217-4227.

[8] Gu Z., B. Witschas, W. van de Water, W. Ubachs, 2013: Rayleigh-Brillouin scattering profiles of air at different temperatures and pressures, Appl. Opt. 52, 4640-4651.

[9] Witschas B., Z. Gu, W. Ubachs, 2014: Temperature retrieval from Rayleigh-Brillouin scattering profiles measured in air, Opt. Exp. 22, 29655-29667.

[10] Reitebuch O., C. Lemmerz, E. Nagel, and U. Paffrath, 2009: The Airborne Demonstrator for the Direct-Detection Doppler Wind Lidar ALADIN on ADM-Aeolus. Part I: Instrument Design and Comparison to Satellite Instrument, J. Atmos. Oceanic Technol. 26, 2501-2515.

[11] Witschas B., C. Lemmerz, O. Reitebuch, 2014: Daytime measurements of atmospheric temperature profiles $(2-15 \mathrm{~km})$ by lidar utilizing Rayleigh-Brillouin scattering, Opt. Lett. 39, 1972-1975. 